\title{
Analytical review of prescription of prophylactic antibiotic in urologic procedures
}

\author{
Mohsen Amjadi-Zeinalhajlou $^{1}{ }^{\circledR}$, Shabnam Mostafazadeh ${ }^{2}$, Elham Jahantabi ${ }^{1}$, Mohsen Mohammad-Rahimi $^{3^{*}}$ \\ ${ }^{1}$ Tabriz University of Medical Sciences, Tabriz, Iran \\ ${ }^{2}$ Department of Urology, School of Medicine, Sina Medical Research and Training Hospital, Tabriz University of Medical \\ Sciences, Tabriz, Iran \\ ${ }^{3}$ Kidney Research Center, Tabriz University of Medical Sciences, Tabriz, Iran
}

\section{Correspondence to:}

Mohsen Mohammad Rahimi, Email:

mm.rahimi1357@gmail.com

Received: 8 Mar. 2021

Accepted: 24 May 2020

ePublished: 11 July 2021

Keywords: Antibiotic, Prophylaxis, Urology, Surgery

\begin{abstract}
Introduction: Prophylactic antibiotics are used before or at the beginning of diagnostic or therapeutic intervention to reduce post-intervention infections.

Objectives: The aim of this study was to compare the administration of prophylactic antibiotics in urological procedures of Imam Reza hospital in Tabriz with the standard protocol.

Patients and Methods: This cross-sectional study was performed on common urological cases including 400 patients who undergo open or endoscopic surgery who received antibiotic prophylaxis before surgery to prevent urinary tract infections.

Results: The mean age of the patients was $49.59 \pm 17.60$ years; $51.5 \%$ of the patients were female. The most common cystoscopy procedure was performed with a frequency of $27.25 \%$ and the most common antibiotic used was cefazolin with a frequency of $34.25 \%$. It was observed that there was a discrepancy between the type of antibiotic in $4.4 \%$ and a discrepancy between the dose of antibiotics used and the standard guideline in $5.5 \%$. The mean duration of drug administration in the studied patients was $3.28 \pm 1.59$ days with a mean of three days. Regarding duration of antibiotic use, in $26 \%$ of cases was contrary to the standard guideline. Additionally, in $11.25 \%$ of cases, the continuation of prophylactic antibiotics in the studied patients was contrary to the standard guideline.

Conclusion: In the present study, the time of administration of prophylactic antibiotics before urological surgeries was the most consistent with the standard guideline. In addition, most cases of discrepancy between dose and type of antibiotic were related to stent replacement and cystoscopy.
\end{abstract}

\section{Introduction}

Urologists use a variety of surgical procedures to treat urological problems (1). Today, urological procedures have changed from open surgery to endoscopic and laparoscopic procedures (2). Infection at the site of surgery and urinary tract infections after surgery are common causes of inability in patients leading to pyelonephritis and other infectious complications $(3,4)$. Prophylactic antibiotics are used before or at the beginning of diagnostic or therapeutic intervention to reduce post-intervention infections (2).

Many inappropriate prophylactic antibiotics are used in various surgeries. Drugs increase the risk of surgical site infection, which requires treatment with broad-spectrum antibiotics that increase the cost and length of hospital stay (5). On the other hand, drug side effects can also be caused by the use of prophylactic antibiotics (2). These include allergic reactions, which

\section{Key point}

In a cross-sectional study on 400 patients who undergo open or endoscopic surgery, who received antibiotic prophylaxis before surgery to prevent urinary tract infections; we found, the time of administration of prophylactic antibiotics before urological surgeries was the most consistent with the standard guideline. In addition, most cases of discrepancy between dose and type of antibiotic were related to stent replacement and cystoscopy.

can range from skin rashes to anaphylactic shock. Besides, prophylactic antibiotics alter the natural flora, which can lead to colitis due to clostridium difficile (5). The effectiveness of prophylactic antibiotics in surgical interventions is determined by patient-related factors, including surgical intervention and the organism causing the infection (3). The antibiotic used should cover the common microorganisms causing the infection in the procedure (6).

Prophylaxis is used only when the benefits

Copyright (C) 2021 The Author(s); Published by Society of Diabetic Nephropathy Prevention. This is an open-access article distributed under the terms of the Creative Commons Attribution License (http://creativecommons.org/licenses/by/4.0), which permits unrestricted use, distribution, and reproduction in any medium, provided the original work is properly cited. 
outweigh the risks and costs, therefore, antibiotics should be prescribed based on high-level studies $(3,6)$. There are protocols for prescribing prophylactic antibiotics in surgical interventions (1).

\section{Objectives}

According to the high prevalence of urological procedures and the fact that there is no standard protocol in Iran, prescribing prophylactic antibiotics in urological procedures is a matter of tact and is not a function of a specific protocol, which reduces the effectiveness of the drug and increases drug resistance, duration of hospitalization, postoperative complications and costs for the health care system and the patient, hence; there is a need for a study that compares prescribed antibiotics with standard protocols to use the results to prescribe antibiotics more effectively. Therefore, the aim of the present study was to compare the administration of prophylactic antibiotics in urological procedures of Imam Reza hospital in Tabriz with the standard protocol.

\section{Patients and Methods}

\section{Study design}

In this cross-sectional study, 400 common urological cases that undergo open or endoscopic surgery and receive antibiotic prophylaxis before surgery to prevent urinary tract infection were included during 2019-2020. Inclusion criteria were hospitalization for urological procedure, negative urine culture at the time of hospitalization, need to prophylactic antibiotics and satisfaction to participate in the study. Exclusion criteria were receiving antibiotics during the last three weeks and, having immunodeficiency diseases, diabetes and age under 18 years.

The information required for this study was extracted from patients' files and entered in the data collection form and compared with the standard protocol of antibiotic prophylaxis in urological surgery. In this way, the researcher referred to the patients' files and extracted the required information and entered in the relevant research checklist consisting of age, gender, weight, reason for hospitalization, type of surgery performed, preoperative diagnosis, postoperative diagnosis, duration of surgery, smoking or addiction, name, dose and time of receiving preoperative antibiotics, name, dose and time of receiving antibiotics after surgery, method of taking antibiotics after discharge, and compliance with standard protocol.

The method of sampling was census which began after the approval of the ethics committee. All patients were included in the study based on inclusion and exclusion criteria and then the frequency of various surgeries was determined. Then patients were grouped based on the type of surgery performed and other study variables were evaluated.

\section{Statistical analysis}

After collecting data, they were statistically analyzed using
SPSS version 18. Independent $t$ test was used to examine the relationship between quantitative variables such as age and compliance of antibiotics with the standard and chisquare test was used to examine the relationship between qualitative variables such as gender. Kolmogorov-Simonov test was used to evaluate the normality of the data. Additionally, to investigate the relationship between age, type of surgery, antibiotic dose and number of antibiotics with the standards based on chi-square coefficient and $P$ value less than 0.05 was considered significant.

\section{Results}

The mean age of the patients was $49.59 \pm 17.60$ years with a median of 49 years. The minimum age of patients was 19 years and the maximum was 79 years. Regarding gender, most of the patients were female (51.5\%). The frequency of the type of surgery performed in the studied patients is shown in Figure 1. As can be seen, the highest frequency is related to cystoscopy.

Comparing the type of antibiotic and the prescribed dose of prophylactic antibiotics in the studied patients with the standard guidelines in the present study, it was observed that there was a discrepancy between the type of antibiotic (4.3\%) and a discrepancy in dose of antibiotic (5.5\%) with the standard guideline (Figures 2 and 3 ).

Comparing the type of antibiotic and the prescribed dose of prophylactic antibiotics in the studied patients with the standard guidelines in the present a discrepancy between the type of antibiotic (4.3\%) and a discrepancy in dose of antibiotic (5.5\%) with the standard guideline were seen. The mean duration of drug administration in the studied patients was $3.28 \pm 1.59$ days with a mean of three days. The minimum time for antibiotic administration was one day and the maximum was seven days. Regarding duration of antibiotic administration, in $26 \%$ of cases in the studied patients had discrepancy with the standard guideline. Accordingly, 11.25\% of case about the time for continuing prophylactic antibiotic had discrepancy with the standard guideline (Figures 2 and 3 ).

\section{Discussion}

The present study showed that in $4.3 \%$ of cases there was a discrepancy between the type of antibiotic with the standard guideline and in 5.5\% of cases there was a discrepancy between the dose of antibiotic used and the standard guideline. Additionally, depending on the type of surgery performed, most of the differences in the type of antibiotic were related to stent replacement and then cystoscopy, respectively. In a study conducted in Melbourne (7) and in a study conducted in Nicaragua (8) prescribing prophylactic antibiotics in the patient who were candidates for surgery was not contrary to the standard protocols. In a study in France, in 53\% of surgical patients from orthopedic, reconstructive, gastrointestinal, urological, and cardiovascular wards, there was a correlation between prophylactic antibiotics and guidelines (9), compared with 


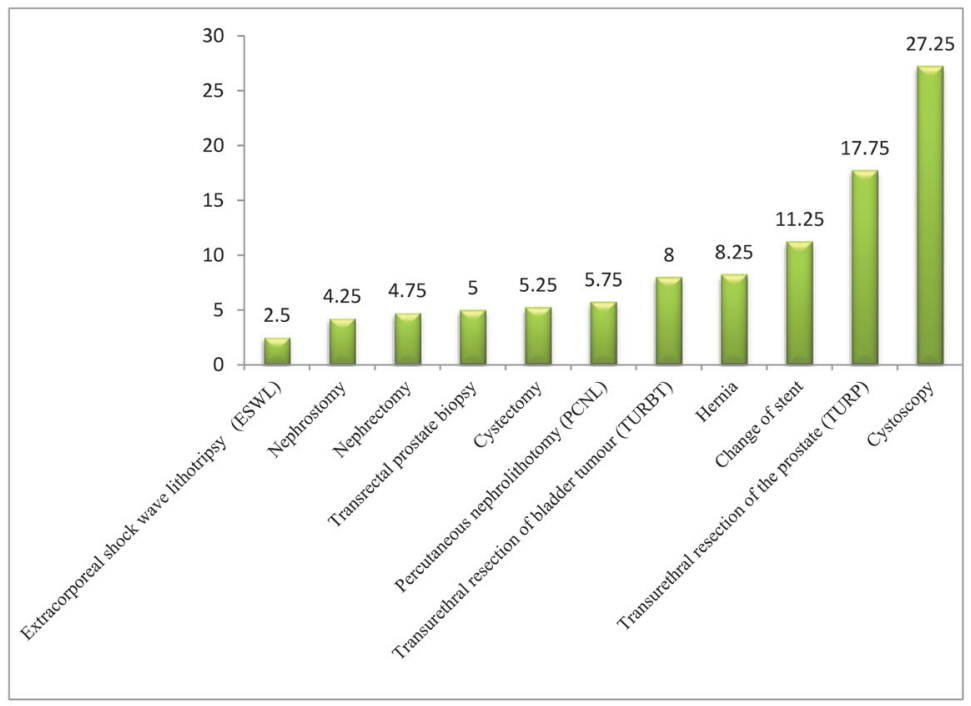

Figure 1. Type of surgery performed in the studied patients

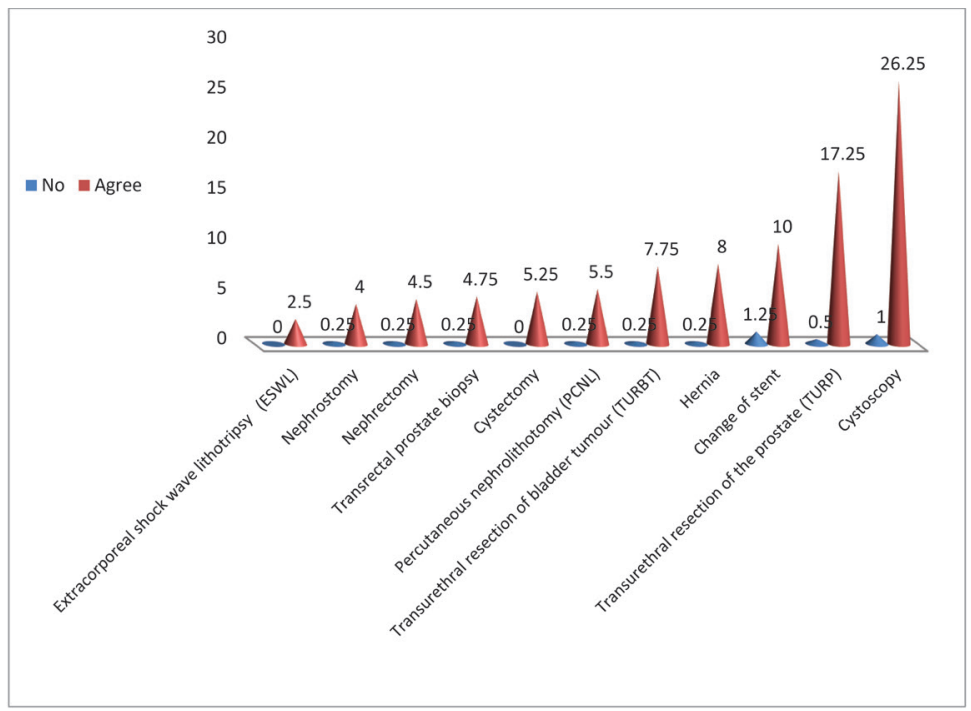

Figure 2. Discrepancy between the type of antibiotic with the standard guideline.

only $19 \%$ in Switzerland. Patients received experimental treatment and only $22 \%$ of patients received inappropriate antibiotics (10). The correct and consistent prescribing of prophylactic antibiotics or discontinuation of antibiotics in our study was highly observed in comparison with other studies, which could be a sign of high awareness of surgical teams about the importance of antibiotics in preventing surgical site infection. This rate was $81 \%$ (11) in study studies in the United States and 84\% in Spain (12). The results obtained in the present study showed that in $11.3 \%$ of cases, the time of administration of prophylactic antibiotics in the studied patients is contrary to the standard guideline. In our study, regarding duration of antibiotic administration, in $26 \%$ of cases in the patients studied, it was contrary to the standard guideline. In a study conducted in Turkey in 2015, consistent with our study,
$40.6 \%$ of cases (13) and in a study conducted in Germany in $41.1 \%$ of cases (14), the duration of prophylactic antibiotics was not appropriate. The results obtained in our study were better and considering about $40 \%$ of the non-compliance with the guideline in the mentioned studies, it seems that surgeons' concern about infection following discontinuation of prophylactic antibiotics leads to a longer duration of antibiotic administration. On the other hand, in our study, there were sterile urological surgeries that can influence the surgeon's opinion about continuing to use antibiotics for a longtime. Disadvantages of overuse and long-term use of antibiotics as prevention include drug toxicity and harmful side effects, increased risk of microbial resistance not only in surgical patients however, in the hospital as a whole and the possibility of super-infection with resistant bacteria and increased costs 


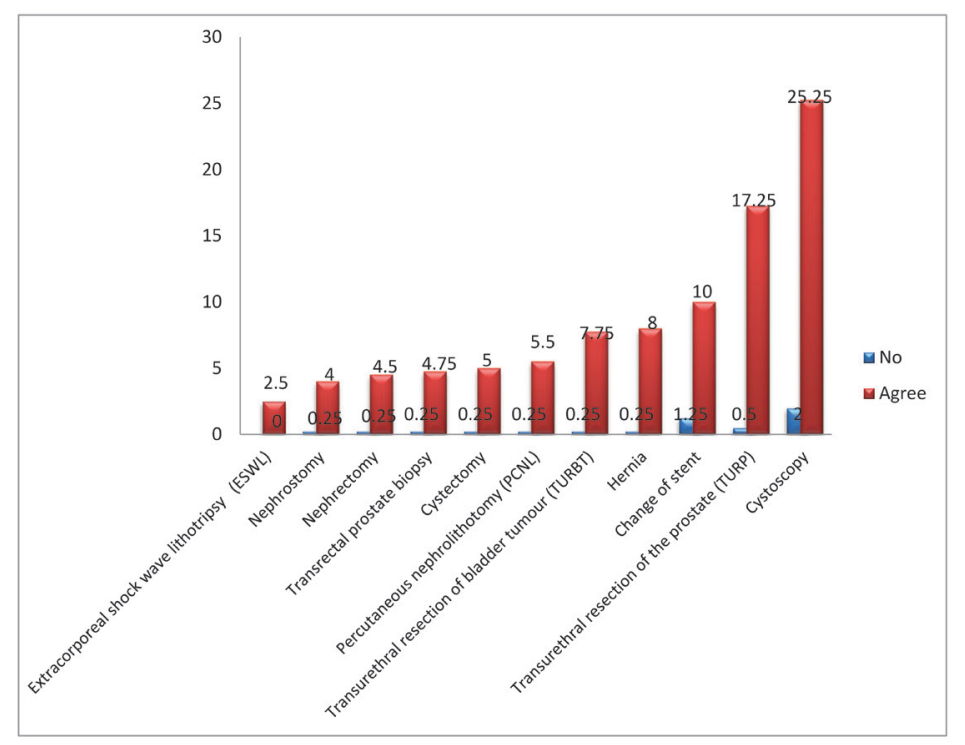

Figure 3. Discrepancy between the dose of antibiotic with the standard guideline.

for the patients and for the hospital that should be seriously considered (15). In the present study, the most common antibiotics used for prophylaxis were cefazolin and then gentamicin + amoxicillin (Table 1). As in previous studies (16), cefazolin was the most common antibiotic. Due to the risk of kidney and ear toxicity following gentamicin use, adherence to the guideline seems necessary. In the present study, more than one type of prophylactic antibiotic was used in a limited number of patients, which was consistent with the guideline compared to similar studies. The use of multiple drugs increases drug interactions, decreases long-term efficacy due to resistance, and increases cost (17). In general, the best method of prevention for most surgeries is intravenous injection. In the present study, intramuscular injection was used for some patients, but it is not suitable for prevention in surgery because it has several pharmacokinetic problems such as slow and unpredictable absorption, which in the end causes low plasma levels of the drug and delay in timely reaching when antibiotics are delivered to the surgical site (18).

\section{Conclusion}

According to the results obtained in this study, it

Table 1. Type of antibiotic used as prophylactic antibiotics

\begin{tabular}{ll}
\hline Antibiotic & Percent \\
\hline Gentamicin + Co-amoxiclav & 2 \\
\hline Tazobactam + piperacillin & 6.25 \\
Gentamicin & 6.75 \\
\hline Ciprofloxacin & 10 \\
\hline Co-amoxiclav & 15.75 \\
\hline Gentamicin+ amoxicillin & 25 \\
\hline Cefazolin & 34.25 \\
\hline
\end{tabular}

can be concluded that in the present study, the time of administration of prophylactic antibiotics before urological surgeries, the type and dose of the drug were most consistent with the standard guideline. Besides, most cases of discrepancy between dose and type of antibiotic were related to stent replacement and cystoscopy.

\section{Limitations of the study}

Generally, the sample size was small. However, further studies with larger sample size are needed to investigate the status of prophylactic antibiotics administration in surgeries.

\section{Authors' contribution}

MA, SM and MMR conducted the research. MMR and EJ collected the data. EJ and SM analyzed the data. MMR prepared the primary draft. All authors edited the final draft and signed the manuscript.

\section{Conflicts of interest}

None.

\section{Ethical issues}

The research followed the tenets of the Declaration of Helsinki. The research was approved by the ethical committee of Tabriz University of Medical Sciences (\#IR.TBZMED.REC.1398.1230). This study was extracted from medical thesis of Shabnam Mostafazadeh (Thesis \# 62216). Accordingly, written informed consent was taken from all participants before any intervention. Moreover, Ethical issues (including plagiarism, data fabrication, double publication) have been completely observed by the authors.

\section{Funding/Support}

The authors would like to thank vice chancellor of research deputy of Tabriz University of Medical Science for supporting this research (Grant\# 62216).

\section{References}

1. Mrkobrada M, Ying I, Mokrycke S, Dresser G, Elsayed S, Bathini V, et al. CUA Guidelines on antibiotic prophylaxis for urologic procedures. Can Urol Assoc J. 2015;9:13-22. doi: 
10.5489/cuaj.2382.

2. Bootsma AM, Laguna Pes MP, Geerlings SE, Goossens A. Antibiotic prophylaxis in urologic procedures: a systematic review. Eur Urol. 2008;54:1270-86. doi: 10.1016/j. eururo.2008.03.033.

3. Luzuriaga Graf J, Sánchez Chavez O, Arauz Arauz E. [Antimicrobial prophylaxis in urologic surgery]. Arch Esp Urol. 1990;43:715-8.

4. Knopf HJ, Graff HJ, Schulze H. Perioperative antibiotic prophylaxis in ureteroscopic stone removal. Eur Urol. 2003;44:115-8. doi: 10.1016/s0302-2838(03)00189-1.

5. Awati SM. Antimicrobial prophylaxis for ureterorenoscopic surgery. Int Surg J. 2016;1:123-7.

6. Kanamaru S, Terai A, Ishitoya S, Kunishima Y, Nishiyama H, Segawa T, et al. Assessment of a protocol for prophylactic antibiotics to prevent perioperative infection in urological surgery: a preliminary study. Int J Urol. 2004;11:355-63. doi: 10.1111/j.1442-2042.2004.00804.x.

7. lerano C, Nankervis JM, James R, Rajkhowa A, Peel T, Thursky K. Surgical antimicrobial prophylaxis. Aust Prescr. 2017;40:2259. doi: 10.18773/austprescr.2017.073.

8. van Disseldorp J, Slingenberg EJ, Matute A, Delgado E, Hak E, Hoepelman IM. Application of guidelines on preoperative antibiotic prophylaxis in León, Nicaragua. Neth J Med. 2006;64:411-6.

9. Bedouch P, Labarère J, Chirpaz E, Allenet B, Lepape A, Fourny $M$, et al. Compliance with guidelines on antibiotic prophylaxis in total hip replacement surgery: results of a retrospective study of 416 patients in a teaching hospital. Infect Control Hosp Epidemiol. 2004;25:302-7. doi: 10.1086/502396.

10. Roger PM, Labate C, Serre S, Zumbo C, Valério L, Bonnet $\mathrm{H}$, et al. Factors associated with effective reassessment of antibiotic therapy on day 3. Med Mal Infect. 2013;43:123-7. doi: 10.1016/j.medmal.2012.12.007.

11. Etienne $\mathrm{P}$, Roger PM, Brofferio P, Labate C, Blanc V, Tiger
F, et al. Antimicrobial stewardship program and quality of antibiotic prescriptions. Med Mal Infect. 2011;41:608-12. doi: 10.1016/j.medmal.2011.07.010.

12. Codina C, Trilla A, Riera N, Tuset M, Carne X, Ribas J, et al. Perioperative antibiotic prophylaxis in Spanish hospitals: results of a questionnaire survey. Hospital Pharmacy Antimicrobial Prophylaxis Study Group. Infect Control Hosp Epidemiol. 1999;20:436-9. doi: 10.1086/501650.

13. Ulu-Kilic A, Alp E, Cevahir F, Tucer B, Demiraslan H, Selçuklu $A$, et al. Economic evaluation of appropriate duration of antibiotic prophylaxis for prevention of neurosurgical infections in a middle-income country. Am J Infect Control. 2015;43:44-7. doi: 10.1016/j.ajic.2014.09.010.

14. Al-Momany NH, Al-Bakri AG, Makahleh ZM, Wazaify MM. Adherence to international antimicrobial prophylaxis guidelines in cardiac surgery: a Jordanian study demonstrates need for quality improvement. J Manag Care Pharm. 2009;15:262-71. doi: 10.18553/jmcp.2009.15.3.262.

15. Sharma M, Eriksson B, Marrone G, Dhaneria S, Lundborg CS. Antibiotic prescribing in two private sector hospitals; one teaching and one non-teaching: a cross-sectional study in Ujjain, India. BMC Infect Dis. 2012;12:155. doi: 10.1186/1471-2334-12-155.

16. Parulekar L, Soman R, Singhal T, Rodrigues C, Dastur FD, Mehta A. How good is compliance with surgical antibiotic prophylaxis guidelines in a tertiary care private hospital in India? a prospective study. Indian J Surg. 2009;71:15-8. doi: 10.1007/s12262-009-0004-9.

17. Ng RS, Chong CP. Surgeons' adherence to guidelines for surgical antimicrobial prophylaxis - a review. Australas Med J. 2012;5:534-40. doi: 10.4066/amj.2012.1312.

18. Zanotto AR, Heineck I, Ferreira MB. Antibiotic prophylaxis in cholecystectomies in a teaching hospital in Brazil. Ann Pharmacother. 2006;40:2003-7. doi: 10.1345/aph.1H165. 